\title{
P153 Radial-finger Pulse Wave Velocity as a Measure of Microvascular Stiffness: Feasibility and Response to Nitroglycerin
}

\author{
Catherine Fortier, Mohsen Agharazii, Charles-Antoine Garneau, Karine Marquis
}

CHU de Québec Research Center-L'Hôtel-Dieu de Québec Hospital, Québec, Canada

\begin{abstract}
Background/Objectives: We previously demonstrated a different regional stiffness response of elastic and muscular arteries after the administration of nitroglycerin, leading to an alteration of aortic-brachial stiffness gradient. Regional stiffness of smaller arteries may respond differently to vasodilation drugs. The objectives of this study were 1- to assess the feasibility of measuring arterial stiffness of the hand, i.e. radial-finger pulse wave velocity (RF-PWV) using piezoelectric sensors, and 2- to measure RF-PWV and its response to nitroglycerin.
\end{abstract}

Methods: This exploratory study was conducted in 11 healthy participants (55\% were men, mean age was $33.6 \pm 10.6$ yrs). Piezoelectric sensors (Complior) were placed simultaneously on carotid and radial arteries, and tip of the third finger, to obtain carotid-radial PWV (CR-PWV) and RF-PWV (in triplicates), before and after 4 minutes of $0.4 \mathrm{mg}$ NTG sublingual spray.

Results: Visually, pressure curves at the finger and detection of the foot of the wave were obtained with a similar quality than radial and carotid pressure curves. The RF-PWV intra-session coefficient of variation was 7.3\%. At baseline, mean CR-PWV and RF-PWV were respectively of $9.07 \pm 1.24 \mathrm{~m} / \mathrm{s}$ and $4.80 \pm 1.42 \mathrm{~m} / \mathrm{s}$. After NTG, CR-PWV decreased $(7.75 \pm 1.32 \mathrm{~m} / \mathrm{s})$ and RF-PWV increased $(6.75 \pm 2.58 \mathrm{~m} / \mathrm{s})$, both significantly $(p<0.01)$.

Conclusion: This first attempt to measure small arteries stiffness shows that it is possible to measure hand PWV. Again, we observed opposite changes in regional stiffness of different vascular territories with a vasodilator drug. These results may open up the path to a better understanding of microcirculation consequences of an altered stiffness gradient.

(c) 2019 Association for Research into Arterial Structure and Physiology. Publishing services by Atlantis Press International B.V.

This is an open access article distributed under the CC BY-NC 4.0 license (http://creativecommons.org/licenses/by-nc/4.0/). 